\title{
Desincentivo a la Investigación: Resultado del Comportamiento Inequitativo del Modelo de Aporte Fiscal Directo (AFD) a las Universidades Chilenas
}

\author{
Patricio E. Ramírez ${ }^{(1)}$ y Jorge L. Alfaro(2) \\ (1) Escuela de Ciencias Empresariales, Universidad Católica del Norte, Larrondo 1281, \\ Coquimbo-Chile. (email: patricio.ramirez@ucn.cl) \\ (2) Departamento de Ingeniería de Sistemas y Computación, Universidad Católica del Norte, \\ Av. Angamos 0610, Antofagasta-Chile. (email: jalfaro@ucn.cl)
}

Recibido Dic. 19, 2011; Aceptado Feb. 09, 2012; Versión final recibida Mar. 29, 2012

\section{Resumen}

El presente trabajo analiza y muestra el comportamiento inequitativo del modelo de Aporte Fiscal Directo (AFD), en el área de investigación científica, que el gobierno chileno otorga a las universidades pertenecientes al Consejo de Rectores de las Universidades Chilenas. Se describe el impacto económico de las publicaciones científicas en el AFD y se determina un valor monetario aproximado de cada publicación, denominado Ingreso Marginal Total (IMT). Además, con el propósito de discutir las implicaciones del modelo AFD como incentivo o desincentivo a la investigación, se analizan los siguientes puntos: (i) el índice de desigualdad de distribución Gini, (ii) la relación entre el IMT y el ranking SCImago, y (iii) la relación entre el IMT la acreditación institucional en investigación. El análisis presentado puede ser de utilidad a las universidades, para, a) auto-control de las unidades, evaluando periódicamente su contribución a los índices de publicaciones, b) desarrollo de políticas de incentivos monetarios por publicación ISI/Scielo, y c) planificación de programas de apoyo a iniciativas científicas.

\section{Disincentive to Research: Result of the Behavioral Inequality of the Direct State Support Model (DSS) to Chilean Universities}

\begin{abstract}
The present work analyzes and shows the behavioral inequality of the Direct State Support (DSS), in the area of scientific research, that the Chilean government grants the universities that belong to the Council of Rectors of Chilean Universities. The economic impact of scientific publications on the DSS and an economic value for each publication, called Total Marginal Revenue (TMR) was determined. Also, in order to discuss the implications of the DSS model as an incentive to research activities, the following points are analyzed: (i) Gini distribution index of inequality; (ii) relationship between TMR and SCImago ranking; and (iii) relationship between TMR and institutional accreditation of research activities. The analyzes presented is useful to universities for the following actions: a) self control of academic units, periodically evaluating their contribution to publication impact; b) to develop university policy that incentivizes ISI and Scielo publications; and c) to plan programs to support research activities.
\end{abstract}

Keywords: scientific publications, direct state support, Gini index, total marginal revenue 


\section{INTRODUCCIÓN}

En una economía basada en el conocimiento, las universidades han llegado a jugar un papel crucial en la competitividad de los países (Cho et al., 2008; Lucchesi, 2011). Estas instituciones actúan como fuente de conocimientos especializados que ayudan a construir ventajas competitivas internacionales para las empresas que los adquieren (Porter, 1998), y al mismo tiempo, ayudan a construir clusters regionales o sectoriales, debido a la abundancia de nuevos conocimientos, permitiendo un importante ahorro en los costos de las actividades empresariales de investigación y desarrollo (Coenen, 2007). Es por ello que fortalecer a las universidades para que desarrollen investigación se está convirtiendo en un tema de agenda nacional, tanto en países desarrollados como en vías de desarrollo (Shin, 2009).

En el contexto mundial, un elemento fundamental en el desarrollo de la actividad científica de las universidades es el aporte financiero estatal (Shibayama, 2011). De hecho, las estrategias de financiación estatal deberían proporcionar un incentivo directo para mejorar el rendimiento de la investigación, y con ello incidir positivamente en un rendimiento económico (Salter y Martin, 2001). En Latinoamérica, existen recientes evidencias de cómo estas estrategias pueden influir positivamente en la conducta de los investigadores (Ubfal y Maffioli, 2011).

Normalmente se utilizan elementos de competencia en el proceso de asignación de fondos públicos a las instituciones de educación superior (Liefner, 2003). Y si bien la literatura concuerda en que no existe una fórmula perfecta para el financiamiento estatal a las universidades (McKeown y Layzell, 1994), es claro que hoy los gobiernos requieren resultados científicos más rápidos y específicos de estas instituciones, como contrapartida a los fondos fiscales entregados para investigación (Tammi, 2009).

En Chile, las universidades son las principales responsables de la actividad científica y generan directamente más del $87 \%$ de la investigación de visibilidad internacional. Y si bien el país ha mostrado un alto nivel de desarrollo económico en los últimos años (Del Sol y Kogan, 2007, PeñaVinces, 2009), existen diversas interrogantes al modelo de crecimiento seguido, y particularmente a los resultados del sistema de educación (Espinoza, 2008; Esquivel-Larrondo, 2007; Matear, 2006). En este contexto, existe una carencia de estudios sobre los efectos de las estrategias de financiación estatal a las universidades chilenas.

Como fruto de un proceso de reformas de los años ochenta (Fried y Abuhadba, 1991), en Chile existen diversas estrategias de apoyo financiero estatal a las universidades. El Aporte Fiscal Directo (AFD) es el instrumento de financiamiento más importante del Estado para las Universidades pertenecientes al Consejo de Rectores de Universidades Chilenas (CRUCH). Este financiamiento es adicional al Aporte Fiscal Indirecto (AFI), el cual se distribuye en función a la matrícula de los alumnos de primer año con los mejores puntajes en la Prueba de Selección Universitaria (PSU), y está dirigido a todas las instituciones de educación superior y no sólo a las pertenecientes al CRUCH (Esquivel-Larrondo, 2007). Adicionalmente el Estado Chileno entrega un Fondo de Desarrollo Institucional (FDI) con el propósito elevar los rendimientos en el uso de recursos de las Universidades pertenecientes al $\mathrm{CRUCH}$. La figura 1 muestra el comportamiento histórico de estos aportes, donde se destaca el incremento del AFD (Fuente: Mineduc).

El AFD se distribuye entre las Universidades del $\mathrm{CRUCH}$ en base a un criterio compuesto de asignación. Este criterio considera que un 95\% de estos recursos se entregan en relación al desempeño histórico del plantel, mientras que un 5\% se hace en relación a índices de desempeño asociados a estadísticas del año anterior: proyectos de investigación y publicaciones, número de jornadas completas equivalentes (JCE) con grados (Magíster o Doctor), número de JCE, número de alumnos de pregrado, y número de carreras de pregrado.

La importancia del incremento/decremento de la fracción del 5\% del AFD que se le asigna a cada institución se debe a que el modelo AFD tiene un efecto recursivo. Tal como explica Wörner(2009) cambios de la fracción del 5\% impactan en la fracción del 95\% del AFD del año siguiente (Para 
una institución particular, si $X_{i}$ es AFD del año i y $A_{i}$ el índice de desempeño de ese año, entonces el AFD para el año $i+1,\left(X_{i+1}\right)$, está definido por: $\left.X_{i+1}=0,95^{\star} X_{i}+0,05^{\star} A_{i}\right)$.

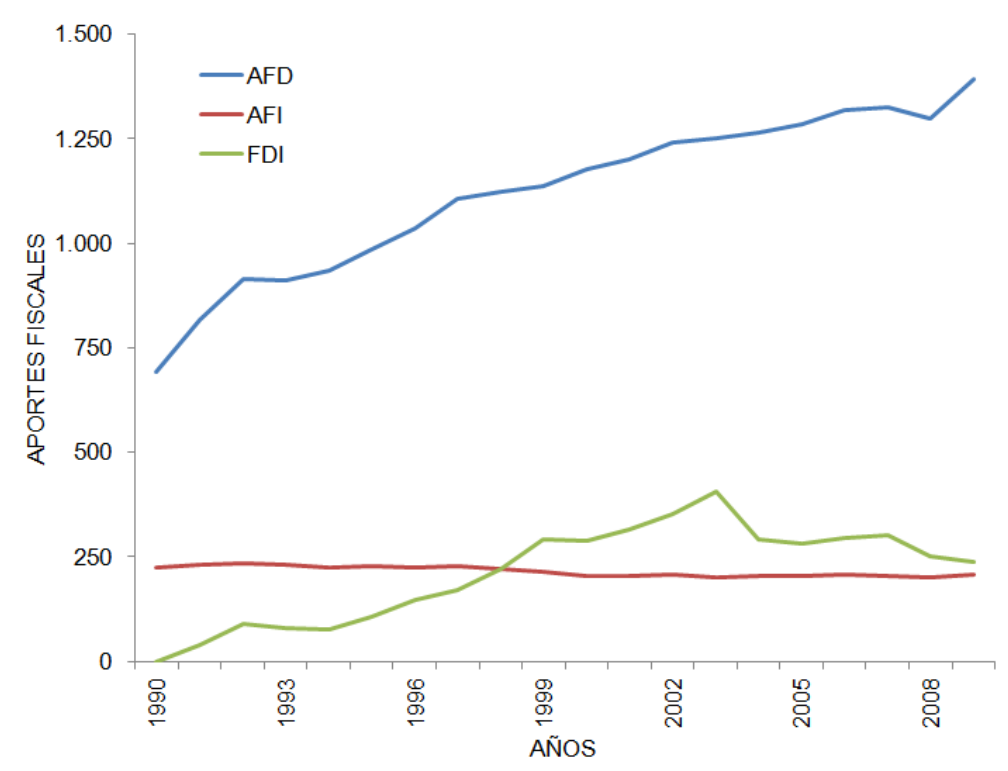

Fig. 1: Aportes fiscales en millones de pesos reales a agosto de 2008.

Ahora bien, más allá de un mecanismo de transferencia financiera ¿ha tenido este modelo de aporte estatal efectos en las instituciones de $\mathrm{CRUCH}$ ? ¿Son estos efectos positivos para el país? El presente estudio tiene como propósito general explorar una dimensión de estos efectos.

En particular, el objetivo de este trabajo es describir el desincentivo a la investigación como resultado del comportamiento inequitativo del modelo de financiamiento AFD para las instituciones del CRUCH.

Para cumplir este objetivo se realizaron dos pasos. Primero, se simuló el modelo AFD para determinar un valor económico aproximado de cada publicación adicional para cada institución del $\mathrm{CRUCH}$, denominado Ingreso Marginal Total (IMT). Segundo, para analizar el comportamiento e implicaciones de la inequidad, se revisó el índice de distribución Gini y las relaciones del IMT tanto con el ranking SCImago como con la acreditación institucional en investigación.

\section{ANTECEDENTES}

Basado en la literatura (Castro, 2000), se puede resumir el modelo que determina la participación en el $5 \%$ del AFD. Tal como se indica en la figura 2, cada año se toman los siguientes indicadores y ponderaciones:

a) Alumnos de pregrado / número de carreras de pregrado (Ponderación: 1\%).

b) Alumnos de pregrado / jornadas académicas completas equivalentes totales (Ponderación: $15 \%)$.

c) Jornadas académicas completas equivalentes con grado académico de magíster y doctor/Jornadas académicas completas equivalentes totales (Ponderación: 24\%).

d) Número de proyectos financiados por el Fondo Nacional de Ciencia y Tecnología (FONDECYT) y otros organismos externos a la universidad / jornadas académicas completas equivalentes totales (Ponderación: 25\%).

e) Número de publicaciones incorporadas a revistas científicas de reconocimiento internacional índice ISI, más un tercio de las publicaciones índice Scielo Chile / jornadas académicas completas equivalentes totales (Ponderación: 35\%).

Luego se aplica un algoritmo de cálculo de seis pasos: 
1. Se obtiene la media y desviación estándar de cada uno de los indicadores básico del conjunto de las universidades.

2. Se realiza un proceso de estandarización, para lo cual a cada valor obtenido en el punto anterior se le resta la media y se divide por la desviación estándar del conjunto de universidades.

3. Se desplazan todos los valores a un tramo positivo, para lo cual se suma dos y se divide por cuatro cada valor anterior.

4. Al valor obtenido en el punto anterior para cada indicador se aplica una función exponencial con la forma e $(x-1.9)^{3}$.

5. El resultado se multiplica por el factor de ponderación de cada indicador, que refleja la importancia relativa de cada coeficiente.

6. Finalmente, se suman los 5 valores obtenidos en cada indicador por cada institución y la participación de este resultado en la suma total de todos los valores de las instituciones es la participación de la institución en el 5\% del AFD.

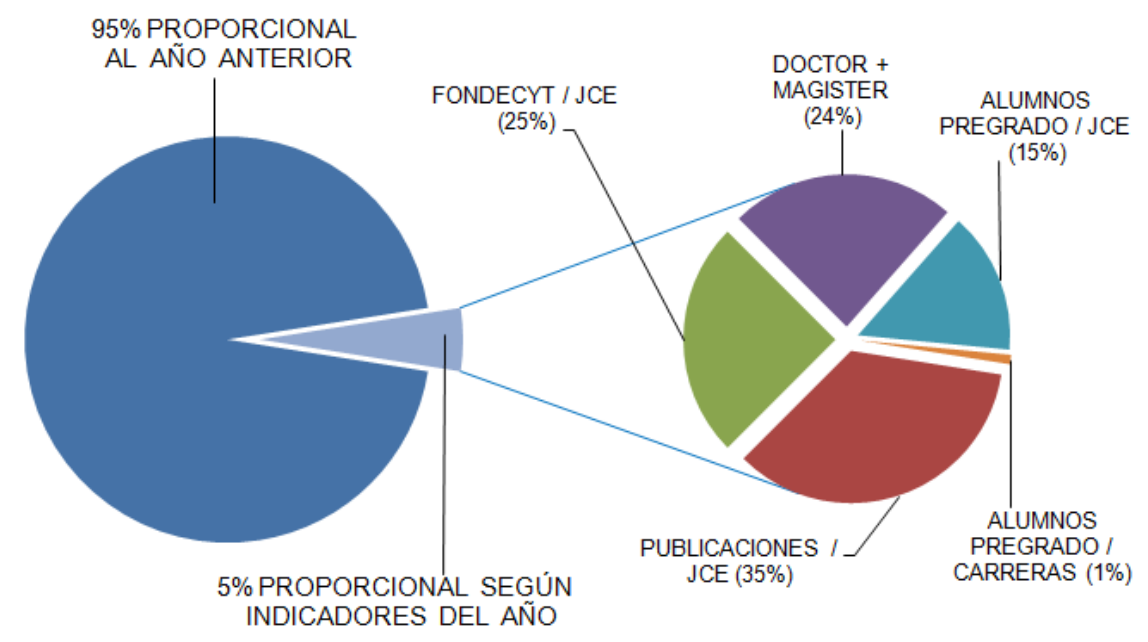

Fig. 2: Recursividad del 5\% del AFD para cada Institución.

El comportamiento del resultado del modelo de asignación del 5\% es exponencial, por tanto, las participaciones entre las universidades de este financiamiento se distribuyen tal como se muestra en la figura 3 ( $X_{k}$ el indicador k-ésimo y $F_{k}$ factor de ponderación del indicador k-ésimo).

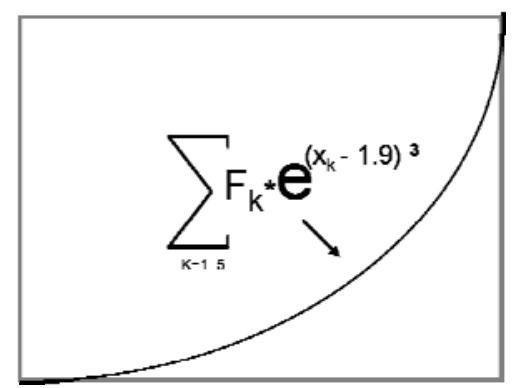

Fig. 3: Resultado del modelo exponencial del 5\% del AFD.

Según la lógica del modelo, se asigna un mejor resultado a mayor distancia de cada indicador con respecto al promedio alcanzado por el conjunto de las instituciones. Por tanto, el avance de una universidad con relación a su historia no tiene impacto real en el resultado si es que esta variación no es mayor que el avance promedio de todas las universidades del consejo de rectores.

Desde una perspectiva global, el indicador de mayor impacto es el número de docentes expresado en jornadas completas equivalentes (JCE). Por lo tanto, una disminución en esta variable genera un aumento significativo en los indicadores y por consiguiente en la participación en el $5 \%$ del AFD y, finalmente, una mayor participación en el total de AFD. 


\section{RESULTADOS}

\section{Modelo simulado AFD}

Se ha simulado un modelo de asignación del 5\% del AFD a partir de los datos públicos para el año 2009 del sistema universitario que recibe este aporte. La figura 4 muestra como la suma de los indicadores por cada institución en el modelo simulado tiene una forma exponencial.

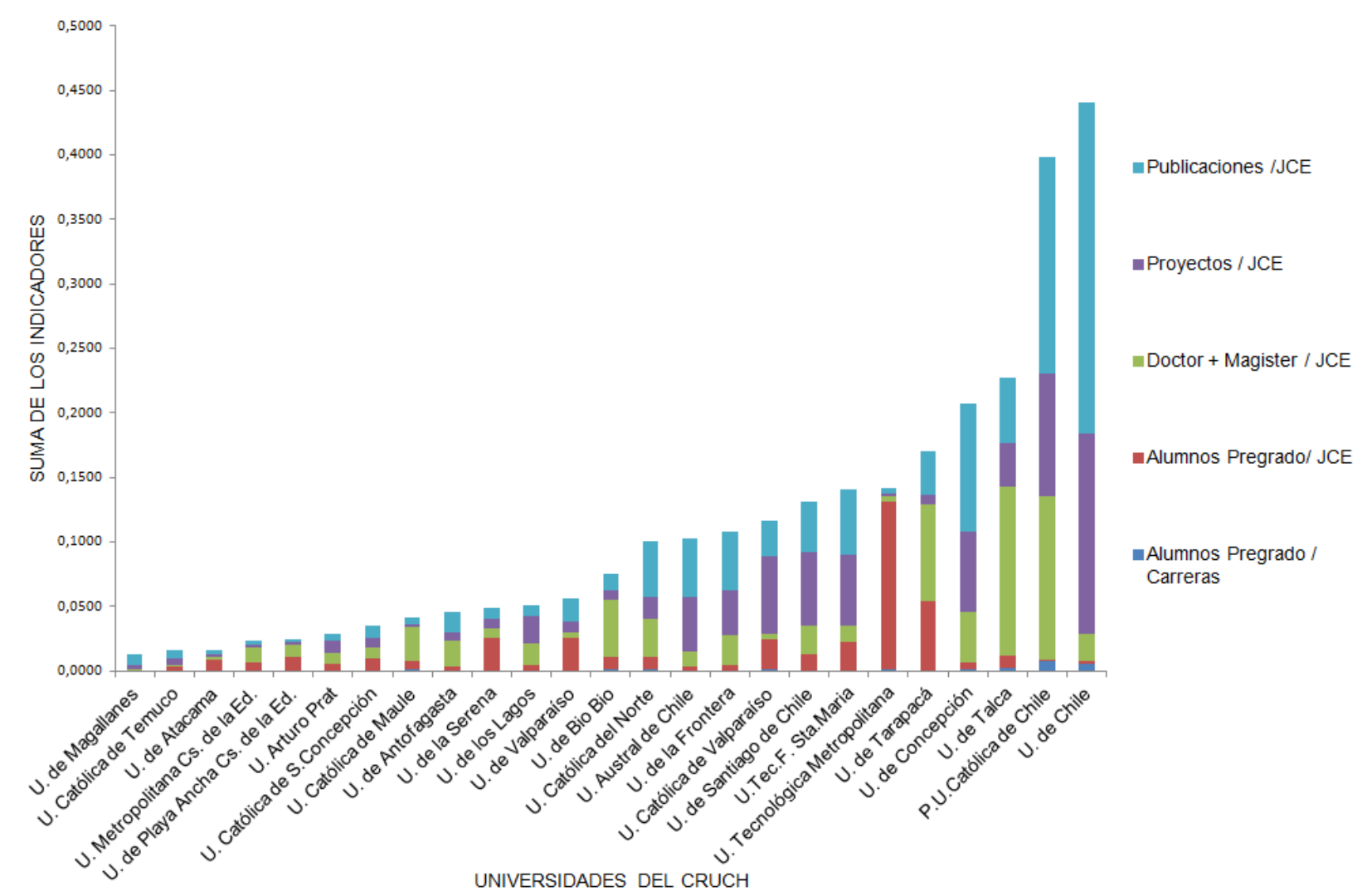

Fig. 4: Suma de los indicadores por cada institución en modelo simulado.

Utilizando el modelo se calculó un valor económico aproximado de una publicación adicional realizada por las universidades del $\mathrm{CRUCH}$. Primero, se determinó un AFD simulado (AFD') que consideró que todas las Universidades aumentaran a la vez una publicación. Luego, a este valor se le resto del AFD del año sin este incremento, calculando con ello el ingreso marginal (IM).

$I M=A^{\prime}-A F D$

El ingreso marginal total (IMT) fue calculado en un horizonte de 5 años, incorporando el efecto de este cambio en los futuros AFD, y con una tasa de descuento del $8 \%$ anual.

$\mathrm{IMT}=\mathrm{IM}+\frac{\mathrm{IM} * 0,95^{1}}{(1+0,08)^{1}}+\frac{\mathrm{IM} * 0,95^{2}}{(1+0,08)^{2}}+\frac{\mathrm{IM} * 0,95^{3}}{(1+0,08)^{3}}+\frac{\mathrm{IM} * 0,95^{4}}{(1+0,08)^{4}}$

La tabla 1 muestra el resultado de este cálculo, los valores se expresan en miles de pesos a Enero de 2010. 
Tabla 1: Valor económico aproximado de una publicación adicional.

\begin{tabular}{lcc}
\hline Universidad & ISI & Scielo \\
\hline U. de Talca & 10.868 & 3.609 \\
U. de Tarapacá & 10.411 & 3.450 \\
U. Técnica F. Santa María & 8.318 & 2.764 \\
U. de la Frontera & 8.245 & 2.739 \\
U. Católica del Norte & 6.945 & 2.308 \\
U. Austral de Chile & 4.627 & 1.539 \\
U. Católica de Valparaíso & 4.454 & 1.480 \\
U. de Concepción & 4.379 & 1.459 \\
U. de Antofagasta & 4.084 & 1.354 \\
U. de Santiago de Chile & 3.671 & 1.221 \\
P.U. Católica de Chile & 3.617 & 1.206 \\
U. Católica de S. Concepción & 3.497 & 1.156 \\
U. de la Serena & 3.246 & 1.073 \\
U. de Chile & 3.127 & 1.043 \\
U. de los Lagos & 3.090 & 1.021 \\
U. de Atacama & 3.017 & 987 \\
U. de Bío Bío & 2.857 & 948 \\
U. de Valparaíso & 2.826 & 939 \\
U. de Magallanes & 2.825 & 935 \\
U. Católica de Temuco & 2.114 & 699 \\
U. Católica de Maule & 2.079 & 686 \\
U. Arturo Prat & 1.920 & 635 \\
U. Metropolitana de Ciencias de la Educación & 1.567 & 517 \\
U. Tecnológica Metropolitana & 1.482 & 490 \\
U. de Playa Ancha de Ciencias de la Educación & 1.054 & 348 \\
\hline & &
\end{tabular}

Análisis de inequidad

El índice Gini (Gini, 1921) mide el nivel de desigualdad de una distribución, su valor varía entre cero y uno. Cero corresponde con la perfecta igualdad (todos los individuos tienen los mismos ingresos) y uno se corresponde con la perfecta desigualdad (un individuo tiene todos los ingresos y los demás ninguno). En estudios anteriores se ha utilizado el índice Gini para analizar desigualdad entre universidades de diversos países (Halffman y Leydesdorff, 2010) o dentro universidades de un único país (Shibayama, 2011). El índice de Gini para esta serie de datos es 0,486, valor coincidente con el reportado para el año 2008 (Wörner, 2009). Sin embargo, este mismo análisis entrega valores de mayor inequidad para los indicadores tomados en forma aislada, tal como se muestra en la Tabla 2, la mayor inequidad es para el indicador Publicaciones IJCE.

Tabla 2: Índices Gini.

\begin{tabular}{lc}
\hline Indicador & Índice de Gini \\
\hline Publicaciones /JCE & 0,620 \\
Proyectos / JCE & 0,601 \\
Doctor + Magister / JCE & 0,582 \\
Alumnos Pregrado/ JCE & 0,604 \\
Alumnos Pregrado / Carreras & 0,611 \\
\hline
\end{tabular}


El SCImago Institutions Ranking (SIR) es un informe mundial que clasifica a más de 2000 de las mejores instituciones de investigación. En la clasificación se incluyen indicadores de rendimiento de investigación, tales como la producción, la visibilidad, la colaboración y el impacto (la tabla 3 muestra las universidades del CRUCH en este ranking). Se examinó si el estar en los dos cuartiles mayores de incentivos era un factor de diferenciación con respecto a aparecer en el SIR. Para este efecto se ejecutó un análisis ANOVA cuyo resultado ( $F=13,905$ y $p=0,001)$ indica que existía tal diferenciación. Del mismo modo, se examinó si el estar en los dos cuartiles mayores de incentivos era un factor de diferenciación con respecto a tener acreditada el área de investigación ante la Comisión Nacional de Acreditación (CNA), ver tabla 4. El resultado del test ANOVA (F = 20,941 y $p=0,000$ ) indica que existe tal diferenciación.

Tabla 3: Universidades del CRUCH en SIR año 2009.

Fuente: http://www.scimagoir.com/

\begin{tabular}{cl}
\hline Ranking & Universidad \\
\hline 384 & Universidad de Chile \\
603 & Pontificia Universidad Católica de Chile \\
859 & Universidad de Concepción \\
1.406 & Universidad de Santiago de Chile \\
1.455 & Universidad Austral de Chile \\
1.663 & Universidad Técnica Federico Santa María \\
1.920 & Pontificia Universidad Católica de Valparaíso \\
2.016 & Universidad Católica del Norte \\
2.038 & Universidad de la Frontera \\
2.115 & Universidad de Valparaíso \\
\hline
\end{tabular}

Tabla 4: Universidades del CRUCH con área de investigación acreditada al 2010.

Fuente: http://www.cnachile.cl

\begin{tabular}{l}
\hline Universidad \\
\hline Pontificia Universidad Católica de Chile \\
Pontificia Universidad Católica de Valparaíso \\
Universidad Austral de Chile \\
Universidad Católica del Norte \\
Universidad de Antofagasta \\
Universidad de Chile \\
Universidad de Concepción \\
Universidad de La Frontera \\
Universidad de Santiago de Chile \\
Universidad de Talca \\
Universidad de Tarapacá \\
Universidad de Valparaíso \\
Universidad del Bío-Bío \\
Universidad Técnica Federico Santa María
\end{tabular}

\section{CONCLUSIONES}

Si bien existe un desarrollo importante en el contexto mundial (Brennan, 2008), en Chile la investigación en gestión de la educación superior es un campo incipiente (Brunner, 2008), este trabajo ha tratado de ser una contribución en ese sentido, aportando elementos cuantitativos al debate nacional sobre el modelo AFD. 
Es un hecho que el desarrollo de la actividad científica de las universidades requiere un significativo aporte financiero de los estados (Shibayama, 2011), como también que las estrategias de financiación estatal tienen efectos en el comportamiento de instituciones e investigadores (Liefner, 2003). Las universidades chilenas no son la excepción a estas reglas, y los hallazgos de este estudio muestran que estos efectos podrían generar y mantener firmes brechas entre las distintas instituciones.

De los resultados de este estudio surge una importante reflexión. ¿Es más valiosa una publicación ISI realizada en, por ejemplo, la Universidad Católica del Norte, que la realizada, por ejemplo, en la Universidad Católica de Temuco? Categóricamente la respuesta es no, y si tuviésemos que detenernos a evaluar la calidad científica de dicha publicación ISI, tendríamos que referirnos a temas como el índice de impacto de la revista donde fue publicada y al número de citas a la publicación, todo ello en el contexto de su disciplina. Lamentablemente, dado la estructura de apoyo financiero del Estado a las instituciones del $\mathrm{CRUCH}$, una publicación ISI puede otorgar beneficios económicos que varían en una relación 1:10 entre la universidad menos favorecida y la más favorecida. También esta inequidad se refleja en el índice Gini para el indicador de desempeño asociado.

Por tanto, es posible que en consecuencia las instituciones con mayor retribución apoyen más la publicación científica, y las otras de menor retribución se concentren en optimizar otros índices de desempeño (tal como alumnos de pregrado /JCE) en desmedro del desarrollo de la ciencia nacional. Por ejemplo, una de línea de análisis posible nos indica que las instituciones con mayores IMT tienden a la excelencia en investigación, es decir, tener sus procesos de investigación más formalizados (acreditados por CNA) y resultados de mayor calidad (ranking SIR). No obstante, por la inequidad expuesta en el modelo, instituciones con bajo IMT, pero con potencial investigador, podrían no proyectarse hacia la excelencia en investigación.

El análisis económico, desde la perspectiva de los beneficios en el 5\% AFD, de los cambios en índices de desempeño es sólo una visión restringida de los efectos tangibles que estos cambios pueden generar en una institución de educación superior perteneciente al CRUCH. Si bien el examen acá presentado tiene la ventaja de cuantificar, en alguna medida, las posibles alternativas de decisión en políticas institucionales en relación a incentivos a los académicos por publicaciones, es claro que debe ser complementado con otras herramientas.

Ahora bien, y a pesar de las restricciones comentadas anteriormente, pensamos que este análisis puede ser de utilidad a las universidades, entre otras cosas para, a) el auto-control de las Facultades/Departamentos/Centros revisando periódicamente su contribución a la formación de los índices de publicaciones, b) desarrollar políticas de incentivos monetarios por publicación ISI/Scielo, o c) presupuestar programas de apoyo a iniciativas científicas.

\section{REFERENCIAS}

Brennan, J., Higher education and social change, Higher Education: 56(3), 381-393 (2008).

Brunner, J.J., El sistema de educación superior en Chile: un enfoque de economía política comparada, Avaliação: 13, 451-486 (2008).

Castro, J., Análisis comparativo del financiamiento a la educación superior, universidades estatales y universidades privadas con aportes 1981-1989 y 1990-1998 (2000), http://www.cedus.cl/files/CASO43.pdf. Acceso: 2 de Diciembre (2011).

Cho, Y., Leem, C. y Shin, K., The relationships among manufacturing innovation, competitiveness, and business performance in the manufacturing industries of Korea, The International Journal of Advanced Manufacturing Technology: 38(7), 840-850 (2008). 
Coenen, L., The role of universities in the regional innovation systems of the North East of England and Scania, Sweden: providing missing links? Environment and Planning C-Government and Policy: 25(6), 803-821 (2007).

Del Sol, P. y Kogan, J., Regional competitive advantage based on pioneering economic reforms: The case of Chilean FDI, Journal of International Business Studies: 38(6), 901-927 (2007).

Espinoza, O., Creating (in) equalities in access to higher education in the context of structural adjustment and post-adjustment policies: the case of Chile, Higher Education:55(3), 269-284 (2008).

Esquivel-Larrondo, J.E., Chile: campo experimental para la reforma universitaria, Perfiles educativos: 29, 41-59 (2007).

Fried, B. y Abuhadba, M., Reforms in higher-education - the case of Chile in the 1980s', Higher Education: 21(2), 137-149 (1991).

Gini, C., Measurement of inequality and incomes, The Economic Journal: 31, 124-126 (1921).

Halffman, W. y Leydesdorff, L., Is inequality among universities increasing? Gini coefficients and the elusive rise of elite universities, Minerva: 48(1), 55-72 (2010).

Liefner, I., Funding, resource allocation, and performance in higher education systems, Higher Education: 46(4), 469-489 (2003).

Lucchesi, Martha A. S., La Universidad Internacional en América Latina: un Nuevo Paradigma para el Siglo XXI, Formación Universitaria: 4(1), 25-36 (2011).

Matear, A., Barriers to equitable access: Higher education policy and practice in Chile since 1990, High Education Policy: 19(1), 31-49 (2006).

McKeown, M.P. y Layzell, D.T. State funding formulas for higher education: Trends and issues, Journal of Education Finance: 19(3), 319-346 (1994).

Peña-Vinces, J.C., Comparative analysis of competitiveness on the Peruvian and Chilean economies from a global view, Journal of Economics, Finance \& Administrative Science: 14(28), 87-105 (2009).

Porter, M.E., On competition. Harvard Business School, Boston, USA (1998).

Salter, A.J. y Martin, B.R., The economic benefits of publicly funded basic research: a critical review, Research Policy: 30(3), 509-532 (2001).

Shibayama, S., Distribution of academic research funds: a case of Japanese national research grant, Scientometrics: 88(1), 43-60 (2011).

Shin, J.C., Building world-class research university: The Brain Korea 21 project, Higher Education: 58(5), 669-688 (2009).

Tammi, T., The competitive funding of university research: the case of Finnish science universities, Higher Education: 57(5), 657-679 (2009).

Ubfal, D. y Maffioli, A., The impact of funding on research collaboration: Evidence from a developing country, Research Policy: 40(9), 1269-1279 (2011).

Wörner, C.H., Aporte fiscal directo para instituciones del Consejo de Rectores de las Universidades Chilenas (1993-2008), Calidad en la Educación: 30, 180-186 (2009). 
